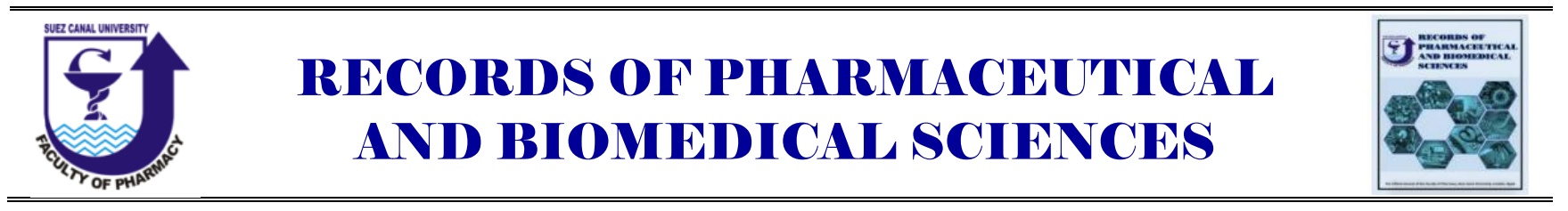

\title{
Nanosponge as a drug delivery system
}

\author{
Norehane Ghorab $^{a}$, Ahmed R. Gardouh ${ }^{\text {b,c }}$, Shaded Gad ${ }^{\text {b* }}$, Yasser Mostafa ${ }^{\mathrm{d}}$ \\ ${ }^{a}$ Medical union pharmaceutical company, Abu Sultan, Ismailia, Egypt, ${ }^{b}$ Department of Pharmaceutics and \\ Industrial Pharmacy, Faculty of Pharmacy Suez Canal University, 41522 Ismailia, Egypt , ${ }^{c}$ Department of \\ Pharmaceutics, Faculty of Pharmacy and Drug Technology, Heliopolis University for Sustainable \\ Development, 2834 El Horreya, Cairo, Egypt ${ }^{d}$ Department of Pharmacology and Toxicology, Faculty of \\ Pharmacy, Suez Canal University, Ismailia, Egypt.
}

Received on: 18.09. 2019

Revised on: 03. 10. 2019

Accepted on: 08. 10. 2019

Correspondence Author:

Tel: +201003934422

E-mail address:

\begin{abstract}
Nano-sponges are colloidal carriers that potentially improve the aqueous solubility of poorly water-soluble drug, retarding its release, improving bioavailability, enhancing physical and chemical stability and decrease skin irritation as well. In addition, hydrogels and Nanosponges are assumed to be excellent applicants for controlled release devices, bio-adhesive devices or targetable devices of therapeutic agents.
\end{abstract}

Keywords: Nanosponge, drug delivery.

\section{Introduction}

Research and development in pharmaceutical industries play an important role in improving, developing and innovating new delivery systems to overcome the problems encountered using conventional delivery systems. This include enhance solubility, increase bioavailability, modify release profile, targeting to specific site, enhance efficacy decrease irritation, improve spread ability, increase stability.

The low water solubility of many drugs is one of the main restrictions to the production of distinct pharmaceuticals. About $40 \%$ of raw medicines in water are badly soluble, hampering their clinical use. Many technological methods have been explored to formulate poorly water-soluble drugs with issues that are hard to fix, including new distribution systems or carriers. (Torchilin, 2000).
The ability of cyclodextrins (CDs) to form inclusion complexes with various molecules is widely used in the pharmaceutical field as a strategy to increase the aqueous solubility and, consequently, bioavailability of lipophilic drugs. For hydrophilic or moderately polar drugs this approach is less effective, and consequently cyclodextrin $(\mathrm{CD})$ derivatives have been investigated.

In attempt to overcome $\beta$-CDs technological limitations as the limited ability to form inclusion complexes, $\beta-\mathrm{CD}$ based Nano-sponges was developed as drug delivery system for encapsulating some drugs, with three-dimensional nanostructure. They are colloidal carriers that potentially improve the aqueous solubility of poorly water-soluble drug, retarding its release, improving bioavailability, enhancing physical and chemical stability and decrease skin irritation as well. In addition, hydrogels and nano-sponges are 
assumed to be excellent applicants for controlled release devices, bio-adhesive devices or targetable devices of therapeutic agents (Peppas et al., 2000).

Although vaginal infections are extremely prevalent, they do not result in high mortality rates. Generally, topical treatment of vaginal infections shown to be effective as oral treatment with higher local drug concentrations and lower adverse effects. The low retention and vaginal discomfort associated with conventional dosage forms motivated scientists to propose new formulation strategies such as the development of mucoadhesive, films, tablets, thermo-gelling systems and nano-technological, to assure high local drug concentrations and minimize systemic drug absorption. It is important that the delivery system and the selected polymers are safe and non-irritating to the mucosa.

The common vaginal infections are caused by bacteria, by fungi, and by protozoa. Disturbances of the natural vaginal milieu have been associated with the occurrence of the more prevalent infections. The ordinary vagina lacks glands, so there is a cervical source for the mucin content of the vaginal fluid. In normal conditions, one expects to find $0.5-0.75 \mathrm{~g}$ of vaginal fluid in the vagina, representing a total daily production of $6 \mathrm{~g}$.

Variations in vaginal fluid amount and composition occur as a consequence of physiological changes (such as age and the menstrual cycle), vaginal practices (such as douching), and by pathological conditions (such as vaginal infections).

The vagina $\mathrm{pH}$ is a protective factor against pathogen, acidic $\mathrm{pH}$ is suitable for Lactobacillus spp. growth with vaginal flora balance for healthy vagina. The Lactobacillus spp. metabolized the glycogen content of the vaginal epithelium to lactic acid, which is an important promoter of the growth of protective flora. Local treatment has been favored over oral approaches in vaginal problems because of the of high local drug concentrations and low drug interactions with the gastrointestinal tract. Vaginal products for the treatment and prevention of vaginal infections are available in more than dosage forms viz. vaginal suppositories, creams, ointments, gels, tablets.

Although, conventional creams, ointments and particularly gels, are more preferable since they easily spread over the vaginal surface area, yet due to the self-cleaning action of the vagina are associated with poor distribution and retention.

\subsection{Complexation}

Complexation is the association between two or more molecules to form a nonbonded entity with a well-defined stoichiometry. Complexation relies on relatively weak forces such as London forces, hydrogen bonding and hydrophobic interactions (Lieberman et al., 1996).

\section{1 a. Stacking complexation}

Stacking complexes are formed by the overlap of the planar regions of aromatic molecules. Nonpolar moieties tend to be squeezed out of water by the strong hydrogen bonding interactions of water. This causes some molecules to minimize the contact with water by aggregation of their hydrocarbon moieties. (Samuel et al., 2007). Some compounds that are known to form stacking complexes as Nicotinamide, Anthracene, Pyrene, Methylene blue, Benzoic acid, Salicylic acid, Caffeine, and Naphthalene etc. (Samuel, 2007).

\section{1. b. Inclusion complexation:}

Inclusion complexes are formed by the insertion of the non-polar molecule (known as guest) into the cavity of another molecule or group of molecules (known as host). The major structural requirement for inclusion complexation is a snug fit of the guest into the cavity of host molecule. The cavity of host must be large enough to accommodate the guest and small enough to eliminate water, so that the total contact between the water and the non-polar regions of the host and the guest is reduced. The most commonly used host molecules are cyclodextrins (Rajewski and Stella, 1996). In this thesis, the effect of complexation with $C D$ on enhancement of dissolution rate will be studied.

\subsection{Cyclodextrins (CDs)}

Cyclodextrins (CDs) are macrocyclic molecules cyclic oligosaccharides synthesized by enzymatic action on hydrolyzed starch. CDs composed of (a1,4)-linked a-D- glucopyranose monomers of the number of glucose units $(6,7$, or 8$)$, named a-, 3-,or g-CDs, respectively (Figure I). They have a characteristic toroidal shape, which forms a welldefined truncated cone-shaped lipophilic cavity. The central cavity is not hydrophobic but considerably less hydrophilic than the aqueous environment and thus can act as a molecular cage for other hydrophobic relatively small molecules, 
while the outer surface is hydrophilic, carrying primary and secondary hydroxyl groups pointing outward (Arun et al., 2008).

Cyclodextrins enhance solubility, dissolution rate and bioavailability of poorly water-soluble drugs by inclusion complexes through non-covalent interactions (Arun et al., 2008). In aqueous solution complexed and uncomplexed drug are in equilibrium (Piel et al., 2006), which shifted towards decomplexation as the free drug is absorbed. CDs increase the amount of drug at the membrane surface, since the drug-CD complexes easily pass aqueous barriers, however, CDs are incapable of permeating through lipophilic membranes and free drug is only able to permeate membranes due to their large size and numerous hydroxyl group on their surface (Arun et al., 2008).

The phase solubility diagram method described by Higuchi and Connors were used to investigate inclusion complexation and assess the solubilization capability of CDs, by plotting substrate solubility as a function of $\mathrm{CD}$ concentration (Figure II). This diagram is classified into type A (the solubility of a substrate increases with increasing ligand concentration over the entire concentration range, indicating the formation of soluble inclusion complex) or type B (a plateau occurs in the curve, indicating the formation of a complex with definite solubility). Type A is subdivided into AL (drug solubility is proportional to CD concentration), AP (positive deviation) and AN (negative deviation). Type B is further classified into a BS (complex with limited solubility) and a $\mathrm{Bi}$ curve (insoluble complex) (Arun et al., 2008; Challa et al., 2005; Tenjarla et al., 1998; Uekama et al., 1998). Tenjarla et al. (1998) discovered that complexes of CDs with miconazole nitrate occurred as an $\mathrm{AL}$ type, except those complexes containing $\alpha$ - and $\gamma$ CD (AN type).

The stability constant and stoichiometry of the complexes can be deducted from the phase solubility diagram (Uekama et al., 1998). The association or stability constant $\mathrm{K}$ can be calculated using the following equation, assuming a 1:1 complex (Challa et al., 2005):

Slope / So (1 - slope)

where: $\mathrm{K}_{1: 1}$ : stability or association constant (M-1)

slope: slope of the linear portion of the stability curve
$\mathrm{S}_{0} \quad$ : intrinsic solubility of the drug under the conditions (M)

The complexation efficiency (CE) for 1:1 complex is defined by the ratio of drug-CD complex to free $\mathrm{CD}$ concentration and can be calculated by using the slope of the curve (Loftsson et al., 2007).

\subsubsection{Production of CDs}

digestion of starch by cyclodextrin glycosyltransferase (CGTase) (Biwer et al., 2002). But the availability of $\mathrm{CDs}$ and high production costs greatly limited their research and application until the 1970's (Horikoshi, 1971). Today, different pharmaceutical products containing CDs are on the market worldwide. More and more CD based dosage forms are underdevelopment (Astakhova and Demina, 2004)

\subsubsection{Chemical Structure of CDs}

CDs are generally crystalline, watersoluble, cyclic, homogeneous, non-reducing, oligosaccharides built up from glucopyranose units. The chemical structure of CDs is shown in figures (III), (IV) and (V). The three commonly used CDs are $\alpha$-CD comprised of six glucopyranose units, $\beta$ $\mathrm{CD}$ comprised of seven units and $\gamma$-CD comprised of eight units. Larger CDs, containing more than eight glucopyranose units in the molecule, have also been studied for their complexation phenomenon (Maestre et al., 2007).

The most important property of CDs is their ability of "entrapping" hydrophobic guest molecules into their cavity. This complexation ability is due to their chemical structure and the glucopyranose units' conformation. In CD molecules, the glucopyranose units are present in the chair conformation. Therefore, the hydroxyl functional groups are orientated to the cone exterior so they have a hydrophilic outer surface. The central cavity is formed by the skeletal carbons and ethereal oxygens of glucose residues, which gives the $\mathrm{CD}$ molecule a comparatively hydrophobic inner cavity (Connors, 1997; Groom et al., 2003). The main driving force for complex formation is thought to be the release of enthalpy rich water from the cavity due to entrapping of guest molecules. Weak Vander Waals forces, hydrogen bonds, and hydrophobic interactions keep the complex together. No covalent bonds are formed during drug-CD complex formation. Therefore, the complexation process can be 

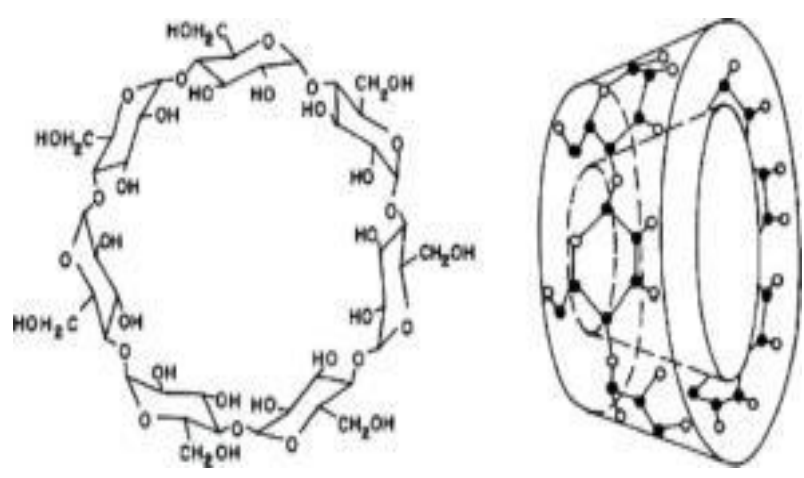

Figure I: Structure of B-Cyclodextrin (Arun et al., 2008)

considered as a replacement of water molecules with drug molecules (Guo et al., 1998; Groom et al., 2003).

Most frequently, the complexation happens between one $\mathrm{CD}$ and one guest (1:1 ratio) molecule. However, 2:1, 1:2, 2:2, and higher order complex equilibriums always exist simultaneously in the system. Phase solubility diagrams are normally used to analyze the complexation stoichiometry. In addition, the complexation is determined both by the CDs' inner cavity size and by the appropriate size of those organic compounds or guest molecules (Szejtli, 1982).

Only those guest molecules with suitable shape and size can be incorporated into the CDs' inner cavity to form inclusion complexes. The cavity size of CDs is dependent on the number of glucoses in the molecule. The cavity size of $\alpha-\mathrm{CD}$ is the smallest of the three CDs and insufficient for many drugs. $\gamma$ $\mathrm{CD}$ has the largest cavity size of all three CDs. However, it is much more expensive than the other CDs. Therefore, $\beta-C D$ is most widely used in research and manufacturing due to its cost and suitable cavity size for most drug molecules (Loftsson, and Brewster, 1996).

The complexation is obviously not suitable for all drugs due to imitation of size and non-polar character of the CD cavity. Drug molecules should fit the following requirements but not without exception to form an applicable complex with $\mathrm{CD}$ (Szejtli, 1998):

- More than 5 atoms (C, P, S, and N) form the skeleton of the drug molecule

- Solubility in water should be less than $10 \mathrm{mg} / \mathrm{ml}$

- Melting point temperature is below $250^{\circ} \mathrm{C}$

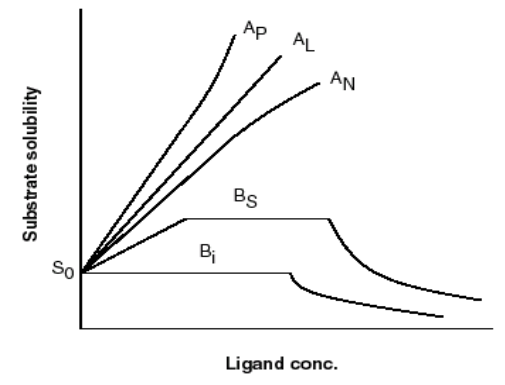

Figure II: Phase Solubility Diagram by Higuchi and Connors, Explaining the Dependence of Substrate Solubility on Ligand Concentration (Arun et al., 2008).

- The molecule consists of less than 5 condensed rings

- Molecular weight between 100 and 400.

This solubilization strategy using CD complexation is not suitable for very small compounds, or compounds that are too large such as peptides, proteins, enzymes, sugars, polysaccharides. However, the side chain in macromolecules may contain suitable groups which can react with CDs in aqueous solutions and form partial complexes with CDs such as insulin (Lovatt et al., 1996).

The kinetics of $\mathrm{CD}$ inclusion complexation has been usually analyzed in terms of a one-step reaction or a consecutive two-step reaction involving intra-complex structural transformation as a second step (Pitha, 1998; Ran et al., 2001).

\subsubsection{Factors affecting complexation with CDs}

\section{- Steric effect}

CDs can interact with a large variety of guest molecules to form non-covalent inclusion complexes as shown in figure (VI). CDs with fewer than 6 units cannot be formed due to steric hindrances while the higher homologous with 9 or more glucose units are very difficult to purify. However, recently, there is isolation and purification method for several kinds of large rings CDs (Uekama et al., 1998). The cavity size of $\alpha-C D$ is insufficient for many drugs and $\gamma-C D$ is expensive. In general, $\delta$-CD has weaker complex forming ability than conventional CDs. $\beta-\mathrm{CD}$ has been widely used because of its ready availability and cavity size suitable for the widest range of drugs (Loftsson, and Brewster, 1996; Martin Del Valle, 2004; Rajewski and Stella, 1996). 


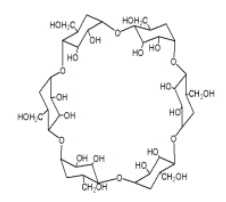

$\alpha \mathrm{CD}$

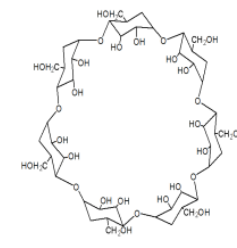

$\beta C D$

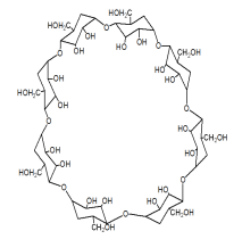

$\gamma \mathrm{CD}$
Figure III Structures of $\alpha-C D, \beta-C D$, and $\gamma-C D$

\section{- Electronic effect}

complexation can be better when the CD and the drug carry opposite charge but may decrease when they carry the same charge. For many acidic drugs forming anions, the cationic (2hydroxy-3-[trimethylammonio] propyl)- $\beta$-CD acted as an excellent solubilizer (Schmid, 1989; Thompson, 1997).

\section{- Temperature}

In most cases, increasing the temperature decreased the magnitude of the apparent stability constant of the drug/CD complex and the effect was reported to be a result of possible reduction of drug/CD interaction forces, such as vander Waals and hydrophobic forces with rise of temperature (Lina and Bär, 2004). However, temperature changes may have negligible effect when the drug/CD interaction is predominantly entropy driven; i.e., resulting from the liberation of water molecules (Bender and Komiyama, 2005).

\section{- Additives}

Adding small amounts of water-soluble polymers or ion pairing agents enhance $C D$ solubilizing effect by increasing the apparent complex stability constant. The polymers or ion pairing agents due to their direct participation in drug complexation, improve both pharmaceutical and biological properties of drug/CD complexes, independent of drug's physiochemical properties (Vromans et al., 1989; Loftsson et al., 2005).

\section{- Co-solvents}

Co-solvents can improve the solubilizing and stabilizing effects of CDs (Pitha and Hoshino, 1992; Stella and Rajewski, 1997).

\section{- Degree of substitution}

The physicochemical properties of CDs, including their complexation ability, may be greatly

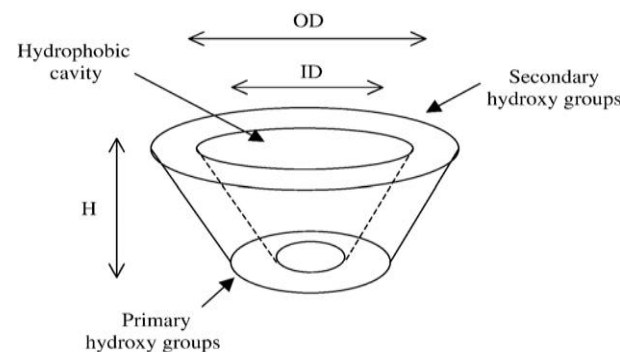

Figure IV Elucidation of Structure of CD molecule.

affected by the type, number, and position of the substituents on the parent CD molecule. When produced under different conditions, the physicochemical properties of HP- $\beta-C D$ samples with same degree of substitution may not be identical owing to the possible occupancy of hydroxypropyl groups at different positions on the parent CD molecule (Connors, 1995). It was reported that increasing the degree of substitution up to an optimum level improves the $\mathrm{CD}$ aqueous solubility, but beyond that, the steric hindrances of the host molecule impair $C D$ complexing (efficiency) capacity. HP- $\beta-C D$ derivatives with a low degree of substitution showed the best complexing properties with low surface activities (Connors, 1997).

\section{- $\mathbf{p H}$}

The effect of various factors such as $\mathrm{pH}$, buffer composition, and addition of different ionic strength adjusters on inclusion complex formation has been reported. For example, the ionization of acidic compounds such as indomethacin and ibuprofen at $\mathrm{pHs}$ above their $\mathrm{pKa}$ values reduces the tendency to complex with CDs. The interference of phosphate buffer and different ionic strength adjusters on the complexation of an azo dye, sodium p-(4-hydroxy-1-naphthylazo) benzenesulfonate with $\mathrm{b}-\mathrm{CD}$ at $\mathrm{pH} 5.9$ was studied by spectrophotometry. The results showed that the complex formation constant was found to increase by increasing phosphate buffer concentration (Omari et al., 2006).

\subsubsection{Method of preparation of drug/CD complex}

Method of preparation as co-grinding, kneading, solid dispersion, solvent evaporation, coprecipitation, spray drying, or freeze drying can affect drug/CD complexation. The effectiveness of a method depends on the nature of the drug and CD. In many cases, spray drying and freeze drying 


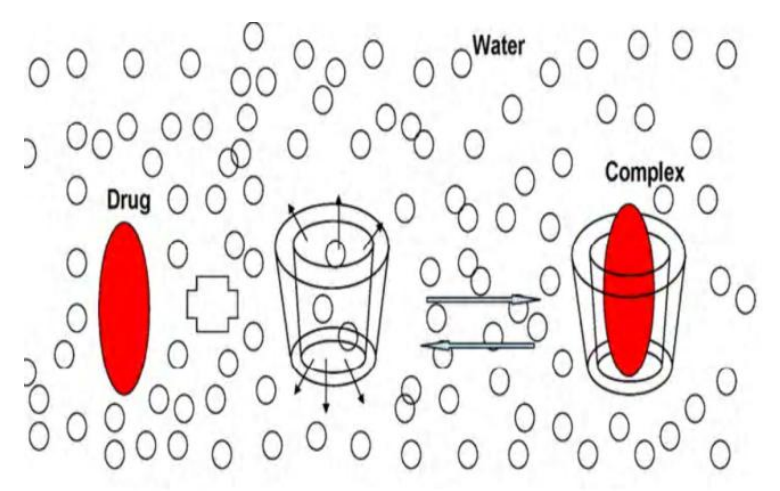

Figure V Complexation process of CDs with drugs

(Szejtli, 1982) were found to be most effective for drug complexation. However, method of preparation showed no influence on the dissolution performance of Tolbutamide: $\beta$-CD complexes (Dressman et al., 1998). Patel et al., (2007) prepared binary system of etoricoxib with $\beta$-CD by the kneading method. The dissolution of etoricoxib was notably increased as compared to pure drug as well as its physical mixture.

\subsubsection{Commercial availability}

The natural CDs, hydroxypropyl (HP), hydroxyethyl (HE), sulfo butyl ether (SBE), and various methylated $C D$ derivatives are available in bulk quantities. Other $\mathrm{CD}$ derivatives are either synthesized in the laboratory for the study or available on laboratory scale (Tomasik and Schilling, 1998).

\subsubsection{CD effects on drug properties in formulation}

\section{- Effect on drug solubility and dissolution}

CDs have been playing a very important role in formulation of poorly water-soluble drugs by improving apparent drug solubility and/or dissolution through inclusion complexation or solid dispersion, by acting as hydrophilic carriers or as tablet dissolution enhancers for drugs with high dose, with which use of a drug/CD complex is difficult, (Loftsson and Brewster, 1996). Reduction of drug crystallinity on complexation or solid dispersion with CDs also contributes to the CD increased apparent drug solubility and dissolution rate. CDs, as a result of their ability to

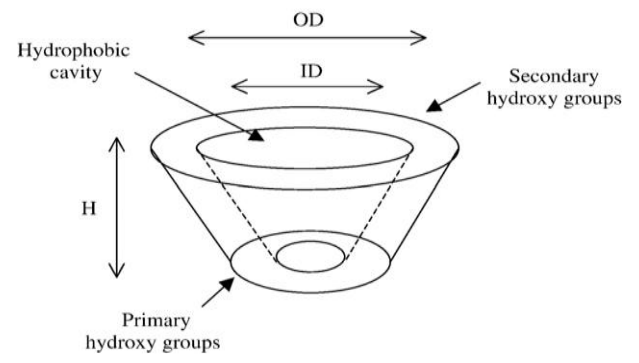

Figure IV Elucidation of Structure of CD molecule.

form in situ inclusion complexes in dissolution medium, can enhance drug dissolution even when there is no complexation in the solid state (AbdelShafi, 2007).

\section{- Effect on drug bioavailability:}

CDs enhance the bioavailability of insoluble drugs by increasing the drug solubility, dissolution, and/or drug permeability. CDs increase the permeability of insoluble, hydrophobic drugs by making the drug available at the surface of the biological barrier, e.g., skin, mucosa, or the eye cornea, from where it partitions into the membrane without disrupting the lipid layers of the barrier. In such cases, it is important to use just enough CD to solubilize the drug in the aqueous vehicle since excess may decrease the drug availability. Addition of polymers can further enhance the drug permeability from aqueous CD solutions (Davies et al., 1998). In the case of water-soluble drugs, CDs increase drug permeability by direct action on mucosal membranes and enhance drug absorption and/or bioavailability (Piel et al., 2006).Unlike detergents, CDs were reported to solubilize membrane components without entering into the membrane, and hence the perturbing effects of CDs can be mild and reversible (Dias et al., 2003).

\section{- Effect on drug safety}

The increased drug efficacy and potency (i.e., reduction of the dose required for optimum therapeutic activity), caused by CD-increased drug solubility, may reduce drug toxicity by making the drug effective at lower doses (Loftsson and Jarvinen, 1999). Further CD entrapment of drugs at the molecular level prevents their direct contact with biological membranes and thus reduces their side effects (by decreasing drug entry into the cells of non-targeted tissues) and local irritation with no 
a)
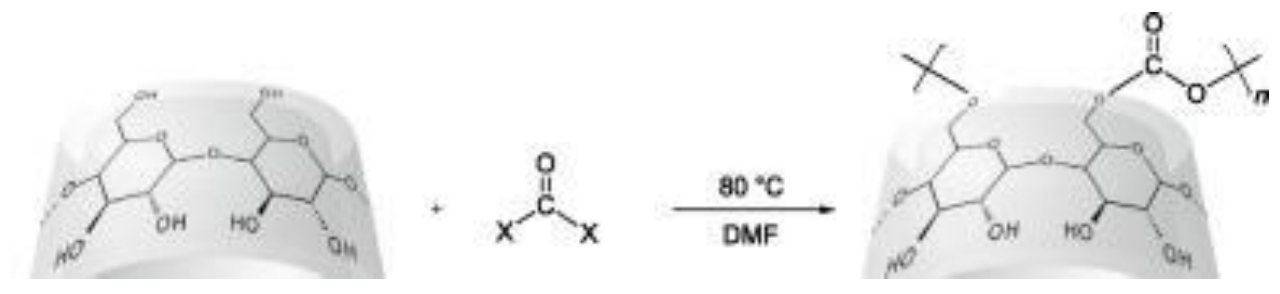

b)
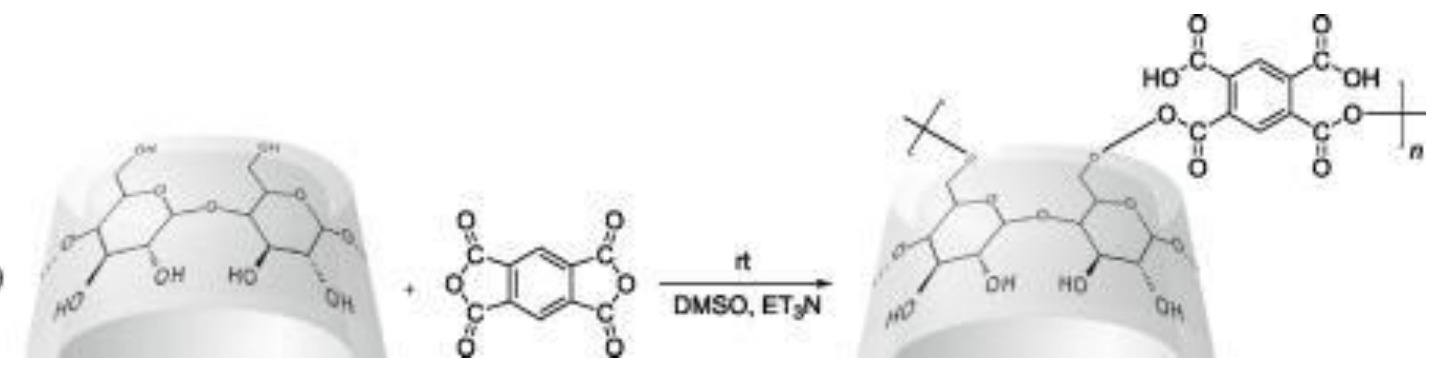

Figure VI Synthetic routes to cyclodextrin Nano-sponges. (a) Cyclodextrin carbonate Nano-sponges. (b) Cyclodextrin carboxylate Nano-sponges

drastic loss of therapeutic benefits (Rajewski and Stella, 1996).

\section{- Effect on drug stability}

CDs can improve the stability of several labile drugs against dehydration, hydrolysis, oxidation, and photodecomposition and thus increase the shelf life of drugs. It was reported that CD-induced enhancement of drug stability may be a result of inhibition of drug interaction with vehicles and/or inhibition of drug bioconversion at the absorption site. By providing a molecular shield, CD complexation encapsulates labile drug molecules at the molecular level and thus insulates them against various degradation processes (Loftsson and Duchêne, 2007).

Since the hydrolysis of drugs encapsulated in CDs is slower than that of free drugs, the stability of the drug/CD complex, i.e., the magnitude of the complex stability constant, plays a significant role in determining the extent of protection (Buschmann et al., 2001).

\section{- Effect on patient compliance}

CDs have also been used to reduce dermal, gastrointestinal, or ocular irritation, mask unpleasant tastes or odors, and prevent adverse drug-ingredient interactions (Loftsson and Jarvinen, 1999) and so, increase patient compliance.

\subsection{Nanospongs}

Nanosponge was originally developed for topical delivery of drugs. They are colloidal carriers have recently been developed and proposed for drug delivery, since their use can solubilize poorly water-soluble drug and provide prolonged release as well as improving drugs bioavailability and modifying its pharmacokinetics parameters. Nanosponges are tiny sponges with a size of about a virus, which can be filled with a wide variety of drugs. The low aqueous solubility $(1.85 \% \mathrm{w} / \mathrm{v}$ at $25^{\circ} \mathrm{C}$ ) of $\beta-\mathrm{CD}$, the cheapest type that is available in pharmaceutical production, and the limited ability to form inclusion complexes with certain molecules, such as hydrophilic or high molecular weight drugs. In an attempt to overcome their limitations and improve their technological characteristics many chemical modifications of CDs have been studied through synthesis of crosslinked CD- based polymers These modified CDs can be obtained by reacting native $\alpha, \beta$ and $\gamma$-CDs with a cross-linking agent such as active carbonyl compound, e.g., carbonyl dimiidazole, triphosgene, diphenyl carbonate, or organic dianhydrides (Scheme 1). The term CD Nano-sponges were first used by De Quan Li and Min Ma in 1998 to indicate a cross-linked $\beta$-CD with organic diisocyanates leading to an insoluble network that showed a very high inclusion constant with several organic pollutants. However, Trotta and co-workers syntheses new kinds of CD Nano-sponges that revealed their full potential in as drug carriers.

Using ultrasound-assisted synthesis and a suitable cross-linker molar ratio, spherical Nano- sponges of submicron size was obtained. The cross-linking produces a powder consisting of cyclodextrin connected by nanochannels to form a cage-like structure. The proposed structure of cyclodextrin Nano-sponges shown in figure VII. 
Table I: Elucidation of some characters of CDs (Uekama et al., 1998)

\begin{tabular}{|c|l|l|c|}
\hline $\begin{array}{l}\text { Type } \\
\text { of CD }\end{array}$ & $\begin{array}{l}\text { Cavity } \\
\text { Diameter }\end{array}$ & $\begin{array}{l}\text { Molecular } \\
\text { Weight }\end{array}$ & $\begin{array}{c}\text { Solubility } \\
(\mathrm{g} / 100 \mathrm{ml})\end{array}$ \\
\hline$\alpha-\mathrm{CD}$ & $4.7-5.3$ & 972 & 14.5 \\
\hline$\beta-\mathrm{CD}$ & $6.0-6.5$ & 1135 & 1.85 \\
\hline$\gamma-\mathrm{CD}$ & $7.5-8.3$ & 1297 & 23.2 \\
\hline$\delta$-CD & $10.3-11.2$ & 1459 & 8.19 \\
\hline
\end{tabular}

Changing the type of CD or amounts of crosslinking agent can modulate the channels between the $\mathrm{CD}$ molecules and the porous network, leading to increase in both the inclusion capacity and the solubilizing ability of the Nano-sponges. Nanosponges surface can be varied from 0.1 to $500 \mathrm{~m}^{2} / \mathrm{g}$ and pore volume range from 0.1 to $0.3 \mathrm{~cm}^{2} / \mathrm{g}$.

Nano-sponges bead can be manufactured by optimizing formulation parameters such as drug: polymer ratio and stirring rate. This results in a large reservoir within each micro sponge, which can be loaded with active agent up to its own weight.

Nano-sponges can be easily dispersed in water and form opalescent stable water suspensions that do not undergo aggregation over time, but do not act as surfactants, with colloidal sizes mean diameter of less than $1 \mu \mathrm{m}$ and a narrow size distribution.

The formed nano-porous structure changes many properties of the native $\mathrm{CD}$ and improves its ability to form specific complexes with guest molecules, that make it suitable for a broad range of applications, that can be either strongly retained or released in a controlled manner. The drugs are dispersed as molecules within the NS structure and

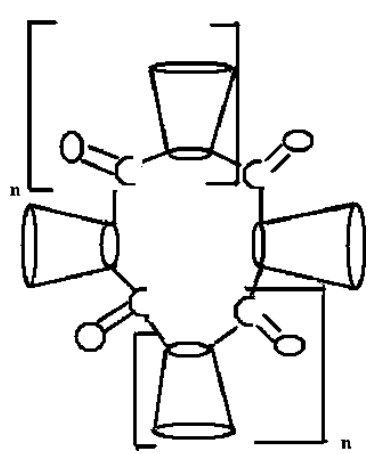

Figure VII: Proposed structure of Nano-sponges of b CD (Swaminathan et al., 2007).

then released as molecules, avoiding the dissolution step. Consequently, many formulation and bioavailability problems can be solved by enhancing the solubility and dissolution rate of the drug. Carbonate Nano-sponges are thermally stable up to $300{ }^{\circ} \mathrm{C}$ and can therefore be sterilized by autoclaving at $121{ }^{\circ} \mathrm{C}$. Degradation of carbonate NS structure was observed under acidic condition $(0.1 \mathrm{~N} \mathrm{HCl})$ at $60^{\circ} \mathrm{C}$ with limited release of $\mathrm{CD}$, whereas stability was not affected under basic condition. Nano-sponges were found to be safe between 500 and $5000 \mathrm{mg} / \mathrm{kg}$ after injection in Swiss albino mice. and with no apparent side effects after oral administration in mice. Nanosponges has been found successful in water treatment from aromatic chlorohydrocarbons, as supports for catalysis applications and in agriculture by encapsulating some important agricultural chemicals with controlled release and slow profile. Nano-sponges have many potential applications in the pharmaceutical field as innovative drug delivery system to enhance solubility, increase stability, and achieve sustained release. They can be used for oral administration as tablets, capsules, pellets, granules, suspensions or solid dispersions and for parenteral administration and for topical administration, by incorporation into topical hydrogel. Table II shows examples for incorporated drugs molecules with different lipophilicities and structures within Nano-sponges structure, either as inclusion complexes or as noninclusion complexes (dexamethasone, flurbiprofen, doxorubicin, itraconazole, resveratrol, paclitaxel, 5fluorouracil and tamoxifen). Formulation of Itraconazole (solubility $1 \mathrm{ng} / \mathrm{mL}$ ) in Nano-sponges improved its solubility more than 27 -fold. Aqueous suspension of $\beta$-CD Nano-sponges enhanced solubilization of the nonsteroidal anti-estrogen Tamoxifen. Nano-sponges enhanced the solubility of poorly soluble difficult to formulate Paclitaxel 
anticancer drug (less than $0.3 \mu \mathrm{g} / \mathrm{mL}$ ). When Paclitaxel loaded in Nano-sponges formed a stable colloidal system in water that inhibited the recrystallization of paclitaxel over time and increased its delivery to cancer cells and lowered its IC50, thereby enhancing its pharmacological effect.

Nano-sponges can be used to design modified release product to provide slow, continuous delivery of the drug over the entire dosing interval. This makes it possible to decrease the dose administered, change the pharmacokinetic profile, and decrease side effects. Flurbiprofen, Doxorubicin were released slowly when incorporated in $\beta$-CD Nanosponges. As well Nelfinavir mesylate, a protease inhibitor with low bioavailability, used to treat HIV infections, loaded Nano-sponges was prepared to enhance the solubility of the drug with slow release from Nano-sponges than from a $\beta$-CD complex.

Nanosponges can also be used to protect encapsulated molecules from light or from chemical and enzyme induced degradation, e.g. encapsulating 5fluorouracile, a light-sensitive drug, in Nanosponges protect it from light degradation, as well shelf life prolongation of camptothecin on encapsulation on Nano-sponges. Nano-sponges also protected the lactone ring from opening due to its high inclusion abilities, thereby increasing stability.

Nanosponges can be used to store and prolong the release of volatile molecules, such as essential oils. Linalool, a liquid component of many essential oils and fragrances with a boiling point of $198{ }^{\circ} \mathrm{C}$, was encapsulated in different types of Nano-sponges as a liquid oil model

The dissolution rate of a solid drug is a limiting factor for oral bioavailability. For hydrophobic drugs the dissolution process acts as the ratecontrolling step and, therefore, determines the rate and degree of absorption. As a consequence, many hydrophobic drugs show erratic and incomplete absorption from the gastrointestinal tract.

The Biopharmaceutics Classification System (BCS) was developed by Amidon in $\mathbf{1 9 9 5}$ as a tool for predicting the extent of drug absorption after oral administration. This system divides drugs into four categories according to their solubility and intestinal permeability. Formulation strategies can be used to shift a drug from one class to another by improving their pharmaceutical characteristics.

Paclitaxel oral bioavailability was increased about 3 fold after administration when loaded in Nano-
Table II Molecules Complexed by Using Nanosponges (Trotta et al., 2012)

\begin{tabular}{|l|l|l|l|}
\hline Dexamethasone & 1.9 & anti-inflammatory & oral, parenteral \\
\hline Flurbiprofen & 4.2 & anti-inflammatory & oral \\
\hline Doxorubicin & 1.3 & antineoplastic & parenter al \\
\hline Progesterone & 3.9 & hormonal & oral \\
\hline Itraconazole & 5.7 & antifungal & oral, topical \\
\hline 5-fluorouracile & -0.9 & antineoplastic & parenter al, topical \\
\hline Tamoxifen & 4.0 & antiestrogen & oral \\
\hline Resveratrol & 2.8 & antioxidant & oral, topical \\
\hline Paclitaxel & 2.5 & antineoplastic & parenter al \\
\hline Camptothecin & 1 & antineoplastic & parenter al \\
\hline Omeprazole & 2.2 & antiulcerative & oral \\
\hline Nelfinavir mesylate & 4.6 & antiviral & oral \\
\hline Acetylsalicylic acid & 1.2 & analgesic & oral \\
\hline Acyclovir & -1.6 & antiviral & oral, topical, parenteral \\
\hline Gamma-oryizanol & - & antioxidant & topical \\
\hline Telmisartan & 7.7 & antihypertensive & oral \\
\hline & & &
\end{tabular}

sponges. The formation of Telmisartan, an antihypertensive BCS class II drug, in NS complex was seen to enhance its dissolution rate.

Nano-sponges can be used in gels or creams for topical application, its ability to increase solubility at the surface of the skin increase the uptake of the guest molecule by the skin. In-vitro studies showed enhance drug permeation of Resveratrol, a polyphenolic phytoalexin from plant sources, on porcine skin and also rabbit buccal mucosa when encapsulated in Nano-sponges. Application of Gamma-oryzanol, a ferulic acid ester mixture, as sunscreen in the cosmetics industry is limited by its high instability and photodegradation, however, its formulation as Nano-sponges in a gel and an $\mathrm{O} / \mathrm{W}$ emulsion showed a good protection from photodegradation.

\subsection{Comparison of Some Effective Vesicular Systems}

Table III shows Comparison of Nanosponge with Vesicular system. Generally, Liposome, niosome, ethosome, transferosome and nanosponge are colloidal drug delivery systems. They all are nanometric in size. Liposome, noisome and transferosomes have some stability problems which is discuss below in table but Nanosponge enhanced the stability of drug.

\subsection{Drug delivery systems for Vaginal Administration}

Traditionally, solutions, suppositories, gels, foams and tablets have been used as vaginal formulations. 
Table III Comparison of Nanosponge with Vesicular system (Kaur et al., 2015).

\begin{tabular}{|c|c|c|c|c|}
\hline \begin{tabular}{|c|} 
Liposome \\
\end{tabular} & \begin{tabular}{|c|} 
Niosome \\
\end{tabular} & Ethosome & Transferosome & \begin{tabular}{|l|} 
Nanosponge \\
\end{tabular} \\
\hline $\begin{array}{l}\text { Liposome consist of one or } \\
\text { more concentric lipid } \\
\text { bilayers, which enclose an } \\
\text { internal aqueous volume. }\end{array}$ & $\begin{array}{l}\text { Niosomes are non- } \\
\text { Ionic surfactant } \\
\text { vesicles obtained on } \\
\text { hydration of } \\
\text { synthetic nonionic } \\
\text { surfactants, withor } \\
\text { without } \\
\text { incorporation of } \\
\text { cholesterol or other } \\
\text { lipids. }\end{array}$ & $\begin{array}{l}\text { Ethosomes are lipid } \\
\text { vesicles containing } \\
\text { phospholipids, alcohol } \\
\text { (ethanol and } \\
\text { isopropylalcohol)in } \\
\text { relatively high } \\
\text { concentration and } \\
\text { water. }\end{array}$ & $\begin{array}{ll}\text { Transferosomes are } \\
\text { vesicular } & \text { system } \\
\text { consisting } & \text { of } \\
\text { phosphatidyl choline and } \\
\text { surfactants. }\end{array}$ & $\begin{array}{l}\text { Nanosponge are } \\
\text { novel class of hyper- } \\
\text { crosslinked polymer } \\
\text { based colloidal } \\
\text { structures consisting } \\
\text { of solid } \\
\text { nanoparticles with } \\
\text { colloidal sizes and } \\
\text { nanosized cavities. }\end{array}$ \\
\hline $\begin{array}{l}\text { The composition of } \\
\text { liposomes is phospholipids } \\
\text { and cholesterol. }\end{array}$ & $\begin{array}{l}\text { They composed of } \\
\text { non-ionic surfactants } \\
\text { and cholesterol. }\end{array}$ & $\begin{array}{l}\text { They composed mainly } \\
\text { of phospholipids, high } \\
\text { concentration of ethanol } \\
\text { and water. }\end{array}$ & $\begin{array}{l}\text { They consist of } \\
\text { phospholipids and } \\
\text { surfactants. }\end{array}$ & $\begin{array}{l}\text { They composed of } \\
\text { polymers and cross } \\
\text { linkers. }\end{array}$ \\
\hline $\begin{array}{l}\text { Stability problems: } \\
\text { due the formation of ice } \\
\text { crystals in liposomes, } \\
\text { the subsequent instability } \\
\text { of bilayers leads to the } \\
\text { leakage of entrapped } \\
\text { material. The oxidation of } \\
\text { cholesterol and } \\
\text { phospholipids also leads to } \\
\text { the formulation instability. }\end{array}$ & $\begin{array}{l}\text { Stability problems: } \\
\text { fusion, aggregation, } \\
\text { sedimentation and } \\
\text { leakage on storage. } \\
\text { The Hydrolysis of } \\
\text { encapsulated drug. }\end{array}$ & $\begin{array}{l}\text { Ethosomes } \\
\text { has initiated a new area } \\
\text { in vesicular } \\
\text { research for transdermal } \\
\text { drug delivery which } \\
\text { can provide better skin } \\
\text { permeation } \\
\text { and stability than } \\
\text { liposomes. } \\
\text { Application of } \\
\text { ethosomes provides the } \\
\text { advantages } \\
\text { such as improved } \\
\text { entrapment and physical } \\
\text { stability. }\end{array}$ & $\begin{array}{l}\text { Stability problem: } \\
\text { chemically unstable } \\
\text { because of their } \\
\text { predisposition } \\
\text { to oxidative } \\
\text { degradation. }\end{array}$ & $\begin{array}{l}\text { Nanosponge } \\
\text { are chemically and } \\
\text { physically stable. } \\
\text { They increase the } \\
\text { stability and } \\
\text { bioavailability, } \\
\text { modify drug release } \\
\text { and reduce side- } \\
\text { effects. }\end{array}$ \\
\hline
\end{tabular}

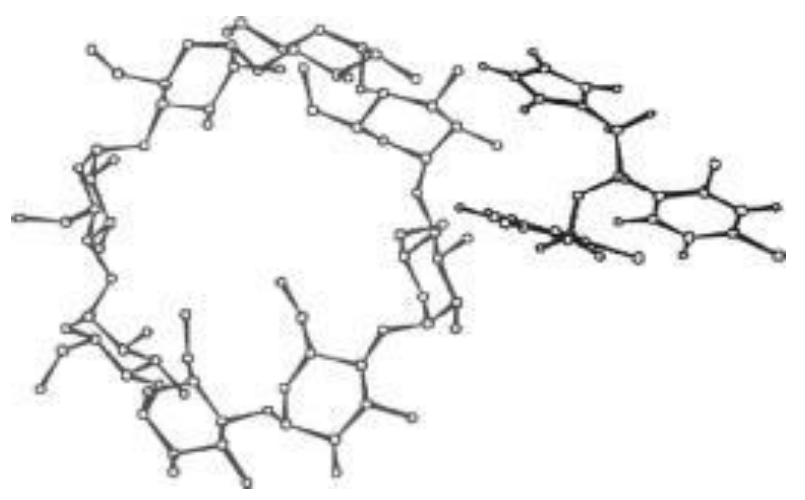

Figure VIII: Structure of Non-Inclusive Complex between Miconazole and $\beta$-CD (LEFT) and the most Probable Miconazole - $\beta$-CD Complex, Showing the Inclusion of Dichlorobenzene -CH-O- of Miconazole (RIGHT) (Piel et al., 2010). 
More recently, vaginal ring has been introduced for hormone replacement and contraceptive therapy.

In general, based on the drug delivery system or formulations used, drug absorption, distribution and residence time in the vagina may vary.

In fact, early work in this field the drug distribution and coverage of vaginal tissue varies considerably with the nature of the delivery system; solution, suspension and foam showing greater superiority over tablet dosage form.

Ideally, a vaginal drug delivery system that is intended for local effect should distribute uniformly throughout the vaginal cavity. Ideally, the choice of vaginal drug administration depends on the applicability of the intended effect; whether a local or topical effect is required. For a local effect to occur, semi-solid or fast dissolving solid system will be required. For a topical effect, generally, a bioadhesive dosage form or intravaginal ring system would be more preferable.

However, by far, it had been difficult to quantitatively measure the distribution of a drug after an intravaginal administration. Vaginal delivery may be designed for the administration of drugs by using an applicator or specifically designed systems for intravaginal administration.

Further, vaginal formulations may be designed to produce local effect such as spermicidal or antibacterial effects or to produce a systemic effect by continuous release of drugs such as contraceptives.

\subsubsection{Creams and gels}

Creams and gels are used for topical delivery of contraceptives and antibacterial drugs. These vaginal dosage forms are messy to apply, uncomfortable and sometimes embarrassing when they leak into the undergarments.

Further, creams and gels may not provide an exact dose because of nonuniform distribution and leakage. In the treatment of bacterial vaginosis, metronidazole and clindamycin vaginal cream are found to be nearly as effective as orally administered drugs.

In the absence of an effective prophylactic anti-HIV vaccine or therapy, current efforts are aimed at developing topical intravaginal formulations of antiHIV agents or microbicides to reduce the mucosal and perinatal virus transmission.
Vaginal creams and gels could be based on the principle of emulsion or hydrogel based drug delivery. During the past few years, considerable work has been done on the development of hydrogel controlled release drug delivery systems. These hydrogels, when placed in an aqueous environment, swell and retain large volumes of water in their swollen structure and release drug in a controlled fashion.

A swelling controlled release hydrogel delivery system for intravaginal administration of an antifungal drug, miconazole, has been reported. Hydrogels are hydrophilic polymers that have been cross-linked by means of covalent bonds.

The vaginal gel has also been used for intravaginal vaccine delivery. Intravaginal delivery of cholera vaccine showed a greater mucosal response in the female genital tract compared to oral administration of the vaccine. Antibacterial agents and drugs for cervical ripening and induction of labor are also available as a vaginal gel form. Oxytocin, dinoprostone and misoprostol are commonly used drugs for cervical ripening and induction of labor.

\subsubsection{Suppositories and Vaginal Tablets}

A large number of vaginal medications are available in the form of tablets or suppositories.

Some authors use the terms pessaries and suppositories interchangeably and consider vaginal tablets as a separate dosage form.

These vaginal formulations are designed to melt in the vaginal cavity and release the drug for several hours. Suppository systems are now most commonly used to administer drugs for cervical ripening prior to childbirth and local delivery of drugs.

Drugs that are administered as suppository include dehydroepiandrosterone sulfate for ripening effect on the uterine cervix, miconazole for vaginal candiasis and progesterone for hormonal replacement therapy.

Vaginal tablets may contain binders, disintegrant and other excipients that are used to prepare conventional oral tablets. It has the advantage of ease of manufacture and insertion. Mucoadhesive polymers are sometimes used in vaginal tablet formulation to increase vaginal residence time.

Drugs that are administered as vaginal tablets 
include itraconazole, clotrimazole and prostaglandins. Presence of hydrophobic and release retarding materials may decrease the absorption of a drug from a vaginal formulation.

Too hydrophobic drugs may not be suitable for vaginal tablets. Presence of penetration enhancers such as surfactants, bile salts can significantly enhance absorption.

\subsubsection{Vaginal rings}

Vaginal rings are circular ring type drug delivery devices designed to release the drug in a controlled fashion after insertion into the vagina. Advantages of vaginal ring are that it is user controlled does not interfere with caution, does not require a daily intake of pills and allows continuous delivery of low dose Steroids. They are approximately $5.5 \mathrm{~cm}$ diameter with a circular cross section diameter of 4$9 \mathrm{~mm}$ and the ring are inserted in the vagina. In simple vaginal rings, drug is homogeneously dispersed within a polymeric ring.

Drug at the surface of the ring is released faster than drug in the inner layer of the ring. Sometimes, drugs in the outermost layer provide an initial burst release. To obtain a constant release of a drug from vaginal ring, sandwich or reservoir type rings has been developed. Sandwich type devices consist of a narrow drug containing layer located below the surface of the ring and positioned between nonmedicated central core and a non-medicated outer band. In reservoir type rings, drugs are dispersed in a centralized core, which is then encapsulated by a drug free layer of polymer.

In a single ring, it is possible to have several cores of different drugs and thereby allowing administration of several drugs from the same device. The rate of drug release can be modified by changing the core diameter or thickness of the nonmedicated coating. The material for making vaginal ring is usually polymeric in nature.

Much of the vaginal ring literature relates to commonly used polymer, poly (dimethyl siloxane) or silicone devices, although other elastomeric polymers such as ethylene vinyl acetate and styrene bartender block copolymer have been tested in recent years.

Ethylene vinyl acetate polymers are classified by the content of vinyl acetate. The addition of vinyl acetate units in the polyethylene provides the following advantages: increased flexibility improved optical properties, greater adhesion, and increased impact and puncture resistance. Further, the clinical acceptability of rings made of ethylene vinyl acetate is very high. Vaginal rings are used for contraceptive and hormone replacement therapy. For most contraceptive applications, the rings are placed in the vagina for 21 days followed by a week of ring free period. NuvaRing is the only combined contraceptive vaginal ring available in the US market.

\subsection{Miconazole Nitrate}

Miconazole (MZ) $\left(\mathrm{C}_{18} \mathrm{H}_{14} \mathrm{Cl}_{4} \mathrm{~N}_{2} \mathrm{O}\right)$ is an imidazole derivative with a broad-spectrum antifungal activity, used as a base or as nitrate $\left(\mathrm{C}_{18} \mathrm{H}_{14} \mathrm{Cl}_{4} \mathrm{~N}_{2} \mathrm{O}\right.$ $\left.\mathrm{HNO}_{3}\right)$. MZ nitrate is only slightly soluble in water $\left(0.17 \pm 0.0002 \mathrm{mg} / \mathrm{mL}\right.$ at $\left.25{ }^{\circ} \mathrm{C}\right), 1$ in 312 of alcohol and 1 in 75 of methanol. MZ is a weak base with a $\mathrm{pKa}$ of 6.7 and with $\mathrm{pH}$ dependent solubility, the lower the $\mathrm{pH}$ the more $\mathrm{MZ}$ is dissolved (Piel et al., 2006; Kovacs et al., 2009).

Miconazole is indicated for superficial candidiasis, dermatophytosis, pytiriasisversi color and disseminated fungal infections (Al-Badr, 2005). It is found to be effective against Microsporum spp. Candida spp., Epidermophyton spp., Trichophyton spp., and Pityrosporonorbiculare (Malassessia furfur). MZ inhibit ergosterol biosynthesis by the inhibition of 14-alpha-demethylase and direct membrane damage to the fungal cell through membrane integrity and fluidity of the fungal cell (Piel et al., 2010; Sawyer et al., 1975).

The main cause of MZ low efficacy in the treatment of systemic mycoses is its limited water solubility. Increasing water solubility improve its oral, parenteral or topical efficacy of antifungal treatment (Tenjarla et al., 1998). The use of various surfactants as adjuvants to overcome the low solubility in formulating antifungal agents in parenteral solutions, unfortunately cause adverse effects such as nausea, vomiting and nephrotoxicity. Many works were done on the use of various different types CDs for complexation of MZ for improving its solubility, in which $\beta-C D$ was found a good vehicle to solubilise MZ in aqueous medium as inclusion complex (Tenjarla et al., 1998). The structure of Complex between Miconazole and $\beta-\mathrm{CD}$ and the most Probable Miconazole - $\beta$-CD Complex, Showing the Inclusion of Dichlorobenzene -CH-O- of Miconazole is shown in figure VIII. 
Table IV Capability of CDs to Form Inclusion Complexes with Miconazole.

\begin{tabular}{|c|c|c|c|}
\hline CD type & Conditions experiment & $\begin{array}{c}\text { Affinity constant } \\
\text { K1:1 (M-1) }\end{array}$ & $\begin{array}{l}\text { Complexation } \\
\text { Efficiency }\end{array}$ \\
\hline & $25 \pm 1{ }^{\circ} \mathrm{C}$ & $333 \pm 18.5$ & \\
\hline \multirow[t]{5}{*}{$\alpha-\mathrm{CD}$} & $0.05 \mathrm{M}$ phosphate buffer $\mathrm{pH} 7.1$, at $23^{\circ} \mathrm{C}$ & $>2.23 \times 106$ & \\
\hline & $25 \pm 1^{\circ} \mathrm{C}$ & $293 \pm 17.6$ & \\
\hline & $0.05 \mathrm{M}$ phosphate buffer $\mathrm{pH} 7.1$, at $23^{\circ} \mathrm{C}$ & $>2.20 \times 105$ & \\
\hline & $\mathrm{pH} 6.0,25^{\circ} \mathrm{C}$ & 97 & \\
\hline & $\mathrm{pH} 7.0,25^{\circ} \mathrm{C}$ & 82 & \\
\hline \multirow[t]{7}{*}{$\beta-\mathrm{CD}$} & $\mathrm{pH} 8.0,25^{\circ} \mathrm{C}$ & 65 & \\
\hline & $\mathrm{pH} 9.0,25^{\circ} \mathrm{C}$ & 39 & \\
\hline & $20^{\circ} \mathrm{C}, \mathrm{pH} 6$ & 117 & \\
\hline & $25^{\circ} \mathrm{C}, \mathrm{pH} 6$ & 96 & \\
\hline & $30^{\circ} \mathrm{C}, \mathrm{pH} 6$ & 85 & \\
\hline & $37^{\circ} \mathrm{C}, \mathrm{pH} 6$ & 63 & \\
\hline & $45^{\circ} \mathrm{C}, \mathrm{pH} 6$ & 41 & \\
\hline \multirow[t]{2}{*}{ methyl- $\beta$ - CD } & $25 \pm 2{ }^{\circ} \mathrm{C}$ & $\begin{array}{l}145.69 \pm 4.1(\mathrm{~K}-1: 1) \\
11.11 \pm 0.5(\mathrm{~K}-1: 2)\end{array}$ & \\
\hline & $25 \pm 2{ }^{\circ} \mathrm{C}$ & $\begin{array}{c}126.94 \pm 4.4(\mathrm{~K}-1: 1) \\
2.20 \pm 0.4(\mathrm{~K}-1: 2)\end{array}$ & \\
\hline \multirow[t]{3}{*}{$\begin{array}{l}\text { 2-hydroxy- } \\
\text { propyl- } \beta-C D\end{array}$} & & & \\
\hline & Ambient temperature & $\begin{array}{l}260 \text { (using So) } \\
55 \text { (using Sint) }\end{array}$ & 0.055 \\
\hline & $25 \pm 1{ }^{\circ} \mathrm{C}$ & $363 \pm 34.1$ & \\
\hline \multirow[t]{2}{*}{$\begin{array}{l}\text { Hydroxyl- } \\
\text { ethyl- } \beta-C D\end{array}$} & $25 \pm 1^{\circ} \mathrm{C}$ & $312 \pm 31.0$ & \\
\hline & $25 \pm 1^{\circ} \mathrm{C}$ & $695 \pm 39.6$ & \\
\hline U-CD & $0.05 \mathrm{M}$ phosphate buffer $\mathrm{pH} 7.1$, at $23^{\circ} \mathrm{C}$ & $>4.30 \times 104$ & \\
\hline $\begin{array}{l}\text { Hydroxyl- } \\
\text { propyly-CD }\end{array}$ & $25 \pm 1^{\circ} \mathrm{C}$ & $305 \pm 27.6$ & \\
\hline
\end{tabular}

MZ-methyl- $\beta-C D$ inclusion complexes were formed using various methods such as coevaporation, spray-drying and lyophilisation; however, the latter method found to be the most effective, economic and easy and delivering the 1.9 highest yield (Ribeiro et al. 2018). Table IV summarizes the CDs inclusion complexes with Miconazole, under different experimental conditions. It is clear that $C D$ could improve MZ efficacy and therapy through CDs complexation that are able to enhance solubility, stability, safety and bioavailability (Arun et al., 2008).

\subsection{Hydrogels}

Hydrogels were designed for the preparation of a mucoadhesive drug delivery system that can reside for extended period of time in a particular area of the body, i.e. increasing the residence time, not only for local targeting of drugs but also for the improved control of systemic drug delivery, reducing the adverse effects and the first pass effect. The most significant goals in the design of mucoadhesive vaginal delivery systems for effective vaginal delivery of antimicrobial agents, 
that the drug delivery system should reside at the site of infection for a prolonged period of time through bioadhesion to the vaginal mucosa, prolonged drug release, improved bioavailability and decreased side effects of the drug and ultimately improved patient compliance. This can be achieved by incorporating mucoadhesive polymers in the formulations for developing and optimizing mucoadhesive gel systems of antifungal drugs for vaginal application.

Wichterle and Lim prepared Hydrogels for the first time in 1960 (Wichterle and Lim, 1960). Hydrogels consist of hydrophilic polymers that form three dimensional networks when on absorbing large amounts of water without dissolving. The formed networks can be composed of a single monomer, or from two or more monomers. It is important to wash out these gels very carefully as most toxicity is related to unreacted monomers, oligomers and initiators. (Peppas et al., 2000).

Hydrogels are biocompatible and quite safe that can be used in many applications, such as tissue

\section{References:}

Abdel-Shafi, A., 2007. Spectroscopic studies on the inclusion complex of 2-naphtol-6-sulfonate with $\beta$ cyclodextrin, Spectrochim. Acta, Part A: Molecular and Biomolecular Spectroscopy, 66, 732-738.

Al-Badr, 2005. Primaquine Diphosphate, Comprehensive Profile, Profiles of Drug Substances, Excipients and Related Methodolog, Harry G. Brittain, Editor, Academic Press, New York, 32: 153-208.

Amidon GL, Lennernäs H, Shah VP, Crison JR. 1995. A theoretical basis for a biopharmaceutic drug classification: the correlation of in vitro drug product dissolution and in vivo bioavailability. Pharm Res. 12(3):413-420.

Arun R, Ashok Kumar CK, Aravanthi VVNSS. 2008. Cyclodextrins as drug carrier molecule: a review article. Sci Pharm 76:567-598.

Bibby, C., Burgess, N., Hill, D., Mustoe, S., 2000. Bird Census Techniques. Academic Press, London.

Buschmann, H., Knittel, D. and Schollmeyer, E., 2001. New textile application of cyclodextrins, J. Incl. Phenom. and Macrocycl. Chem., 40, 169-172. engineering (in artificial hearts and artificial skin), contact lenses, drug delivery devices, membranes for biosensors, (Peppas et al., 2000).

Drug release through hydrogels as drug delivery systems can be controlled by diffusion, swelling or chemically. Hydrogels polymer composition, water content, crosslinking density and crystallinity, amount of incorporated drug, and drug-polymer interactions can affect drug release rates, duration and dissolution profiles (Peppas et al., 2000; Lin and Metters, 2006; Kim et al., 1994; Satish et al. 2006).

The limited affinity of the poorly water-soluble drugs for the hydrophilic hydrogels can be enhanced by using CDs in gels to enhance drug solubility, entrapment, and control drug release Bibby et al. (2000).

However, the polymer swelling, and drug release rate might be restricted by complex formation between drug and $\mathrm{CD}$ as result of decrease in network mesh size (Bibby et al., 2000).

Davies, D., Deary, M. and Wealleans, D., 1998. Stability constants of $\alpha$-cyclodextrin complexes of para-substituted aromatic ketones in aqueous solution, J. Chem. Soc., Perkin Trans. (2) 193196.

Dias, M., Raghavan, S., Pellett, M. and Hadgraft, J., 2003. The effect of $\beta$-cyclodextrin on the permeation of diclofenac from supersaturated solutions, Int. j. Pharm., 263, 173-181.

Dressman, J., Amidon, G., Reppas, C. and Shah, V., 1998. Dissolution testing as a prognostic tool for oral drug absorption: immediate release dosage forms, Pharm. Res., 15, 11-22.

Kaur, G., Narang, R., Rath, G. \& Goyal, A. K. 2012. Advances In Pulmonary Delivery Of Nanoparticles. Artificial Cells, Blood Substitutes, And Biotechnology, 40, 75-96.

Kim, C.K., Kim, M.J., Oh, K.H., 1994. Preparation and evaluation of sustained release microspheres of terbutaline sulfate. Int. J. Pharm. 106, 213-219.

Kovacs G1, Berghold A, Scheidl S, Olschewski H, 2009. Pulmonary arterial pressure during rest and exercise in healthy subjects: a systematic review. Eur Respir J. 34(4):888-94. 
Lin, C. C., and Metters, A. T. 2006. Hydrogels in controlled release formulations: network design and mathematical modeling. Advanced Drug Delivery Reviews, 58(12e13), 1379e1408.

Loftsson, T. and Duchêne, D., 2007. Cyclodextrins and their pharmaceutical applications, Int. J. Pharm., 329, 1-11.

Loftsson, T. and Jarvinen, T., 1999. Cyclodextrins in ophthalmic drug delivery, Adv. Drug Deliv. Rev., 36 (1) 59-79.

Patel, R., Patel, M. \& Suthar, A. 2007. Spray Drying Technology: An Overview. Indian Journal Of Science And Technology, 2, 44-47.

Peppas,P., Bures,W., Leobandung Hideki, Ichikawa Hideki Ichikawa Pham, D.-D., Fattal, E. \& Tsapis, N. 2015. Pulmonary Drug Delivery Systems For Tuberculosis Treatment. International Journal Of Pharmaceutics, 478, 517-529.

Piel FB, Patil AP, Howes RE et al. 2010. Global distribution of the sickle cell gene and geographical confirmation of the malaria hypothesis. Nature Communications 1, 104.

Piel, G., Piette, M., Barillaro, V., Castagne, D., Evrard, B. and Delattre, L., 2006. Bethamethasonein-cyclodextrin-in-liposome: the effect of cyclodextrins on encapsulation efficacy and release kinetics, Int. J.Pharm., 312, 75-82.

Rajewski, R. and Stella, V., 1996. Pharmaceutical applications of cyclodextrins, II: in vivo drug delivery,J. Pharm.Sci., 85, 1142-1169.

Ribeiro, A.R., Sures, B., Schmidt, T.C., 2018. Cephalosporin antibiotics in the aquatic environment, a critical review of occurrence, fate, ecotoxicity and removal technologies. Environ. Pollut. 241, 1153-1166.

Satish U, Cleckner L, Vasselli J. 2006. Pilot study of using strategic management simulation to assess human productivity. In: AWMA/EPA Conference: Indoor Air Quality - Problems, Research, and Solutions, Durham, NC, US.

Sawyer, R.T., A.R. Lawler and R.M. Overstreet. 1975. Marine leeches of the eastern United States and the Gulf of Mexico with a key to the species. Journal of Natural History 9: 633-667

Swaminathan P. R., Vavia Francesco, Trotta Satyen Torne. 2007. Formulation of betacyclodextrin based nanosponges of itraconazole. J Incl Phenom Macrocycl Chem , 57:89-94

Szejtli, J., 1982. Cyclodextrins and their inclusion complexes, Akademiai Kiadó, Budapest, 204-232.

Tenjarla, S., Puranajoti, P., Kasina, R., Mandal, T., 1998. Preparation, characterization, and evaluation of miconazole-cyclodextrin complexes for improved oral and topical delivery. J. Pharm. Sci. $87,425-429$.

Tomasik, P. and Schilling, C. 1998. Complexes of starch with inorganic guests,in: D. Horton Publishers, Advances in carbohydrate chemistry and biochemistry, Academic Press, San Diego, 263-343.

Trotta, Marco Zanetti \& Roberta Cavalli. 2012. Cyclodextrin-based nanosponges as drug carriers. Beilstein J. Org. Chem., 8, 2091-2099.

Wichterle, O. And Lím, D. 1960. Hydrophilic Gels for Biological Use. Nature 185, 117-118. 